\title{
Drug Reaction with Eosinophilia and Systemic Symptom Syndrome Induced by Lamotrigine
}

\author{
Song Hee Han, Min Seok Hur, Hae Jeong Youn, Nam Kyung Roh, Yang Won Lee, Yong Beom Choe, \\ Kyu Joong Ahn
}

Department of Dermatology, Konkuk University School of Medicine, Seoul, Korea

Drug reaction with eosinophilia and systemic symptom (DRESS) syndrome is a type of severe adverse drug-induced reaction. Dermatologists should make a quick diagnosis and provide appropriate treatment for DRESS syndrome to reduce mortality rates, which can be as high as $10 \%$. We present the case of a 47-year-old man with schizoaffective disorder treated with lamotrigine who developed DRESS syndrome to emphasize the importance of close observation of patients with drug eruption. He was consulted for erythematous maculopapular rashes on the trunk that developed 3 weeks after starting lamotrigine. A few days later, he developed generalized influenza-like symptoms. The skin rashes spread over his entire body, and the sense of itching was rapidly aggravated within a few days. Increased liver enzyme levels and significant eosinophilia were found on laboratory test results. His condition was diagnosed as DRESS syndrome, and he was treated with systemic and topical corticosteroids for 2 weeks. (Ann Dermatol 29(2) 206 209, 2017)

\section{-Keywords-}

Anticonvulsants, Drug hypersensitivity syndrome

Received April 11, 2016, Revised June 20, 2016, Accepted for publication July 15, 2016

Corresponding author: Kyu Joong Ahn, Department of Dermatology, Konkuk University School of Medicine, 120-1 Neungdong-ro, Gwangjin-gu, Seoul 05030, Korea. Tel: 82-2-2030-5172, Fax: 82-2-2030-5179, E-mail: kjahn@ kuh.ac.kr

This is an Open Access article distributed under the terms of the Creative Commons Attribution Non-Commercial License (http://creativecommons. org/licenses/by-nc/4.0) which permits unrestricted non-commercial use, distribution, and reproduction in any medium, provided the original work is properly cited.

Copyright $($ c The Korean Dermatological Association and The Korean Society for Investigative Dermatology

\section{INTRODUCTION}

Drug reaction with eosinophilia and systemic symptom (DRESS) syndrome is a type of rare severe drug eruption, and it along with Stevens-Johnson syndrome and toxic epidermal necrolysis can even be life-threatening ${ }^{1}$. The major characteristics of DRESS syndrome include skin rashes combined with a fever, internal organ involvement, and eosinophilia on laboratory test results. The liver is most commonly involved, and in some cases, the kidney, lung, or nervous system is affected ${ }^{1,2}$. It has been reported that drugs that may cause DRESS syndrome are usually aromatic anticonvulsants (e.g., carbamazepine, phenytoin, and phenobarbital), sulfonamides, and allopurinols ${ }^{3-6}$. During the last 10 years, as the use of lamotrigine, a new aromatic anticonvulsant, has increased, reports on cases with DRESS syndrome have also increased ${ }^{1,7-9}$. We report a case of DRESS syndrome induced by lamotrigine treatment and a review of the literature.

\section{CASE REPORT}

A 47-year-old Korean man presented with the complaint of itchy skin rashes over his entire body. He was consulted in the department of dermatology for maculopapular skin rashes that developed during hospitalization in the psychiatric ward. He had been taking antipsychotic drugs, including quetiapine, aripiprazole, and risperidone, for schizoaffective disorder, and lamotrigine was added 3 weeks before the outbreak of rashes. As the clinical impression was drug eruption induced by lamotrigine, a new aromatic anticonvulsant, we suggested discontinuing this drug and observing the patient's condition over time. Two days later, he had a fever over $38^{\circ} \mathrm{C}$, the skin rashes that had been confined to the trunk spread to his entire body, 
and his sense of itching became aggravated. The patient re-consulted with the department of dermatology. He showed erythematous maculopapular rashes disseminated over his entire body, and the rashes combined with each other as they progressed. Also, there was significant involvement of the face with erythematous edema, and oral erosions and rubor were observed (Fig. 1).

Laboratory test findings revealed absolute eosinophilia (white blood cells, $7.46 \times 10^{9} \mathrm{~L}^{-1}$; neutrophil segmented, $47 \%$; neutrophil band, $5 \%$; lymphocytes, $12 \%$; monocytes, $7 \%$; eosinophils, $28 \%, 2.09 \times 10^{9} \mathrm{~L}^{-1}$; basophils, $1 \%$ ), and mildly elevated liver enzymes (aspartate aminotransferase [AST], $37 \mathrm{U} / \mathrm{L}$; alanine aminotransferase [ALT], $66 \mathrm{U} / \mathrm{L}$; alkaline phosphatase [ALP], $148 \mathrm{U} / \mathrm{L}$; total bilirubin, $1.43 \mathrm{mg} / \mathrm{dl}$ ). A 4-mm punch biopsy of the above-knee lesion was obtained. Hematoxylin and eosin staining showed mild alteration of the epidermis, including vacuolar degeneration of the basal cell layer. Exocytosis of the lymphocytes was observed at the dermo-epidermal junction. In addition, interstitial or perivascular infiltration of lymphocytes and histiocytes, as well as some eosinophils in the dermis, were significant (Fig. 2). This indicated superficial vacuolar interface dermatitis, suggesting a diagnosis of drug eruption.

The patient's condition was diagnosed as DRESS syndrome, a severe drug eruption that is accompanied by a fever, eosinophilia, and internal organ involvement. Accordingly, we started systemic (methylprednisolone $65 \mathrm{mg}$ IV, q 12 hours) and topical (methylprednisolone aceponate, $0.1 \%$ ) steroid treatment immediately. He showed remarkable improvement during the next 2 weeks, and the levels of eosinophils and liver enzymes normalized. Subsequently, we gradually decreased the dosage of systemic corticosteroids and maintained the patient on an antihistamine. Ultimately, he had remarkable clinical im- provements in the skin rashes, and the sense of itchiness almost disappeared.

Informed consent was obtained from the patient prior to this report.

\section{DISCUSSION}

Drug eruption refers to skin rashes caused by any kind of drug. Similar to the diversity of its clinical features, its severity varies from simple rashes to the involvement of internal organs. It has a relatively low incidence of 1 in every 1,000 or 10,000 persons worldwide ${ }^{10-12}$. Symptom outbreak after an average of 2 to 8 weeks after the administration of the causative drug and a prolonged silent peri-

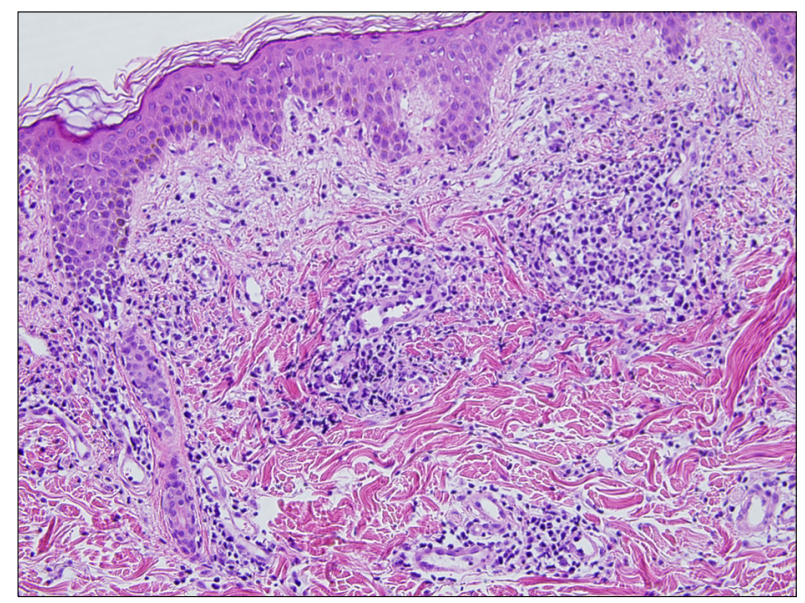

Fig. 2. A 4-mm punch biopsy specimen showing vacuolar degeneration of the basal cell layer and mild spongiosis of the epidermis, exocytosis of the lymphocytes at the dermo-epidermal junction, and interstitial or perivascular infiltration of lymphocytes and histiocytes as well as some eosinophils in the dermis (H\&E, $\times 200$ ).
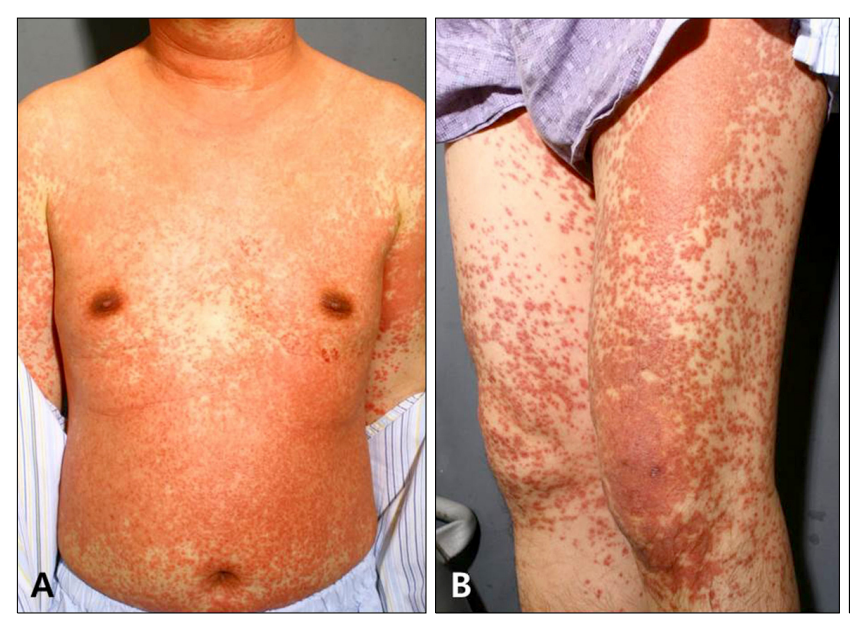

Fig. 1. (A, B) Disseminated, erythematous, maculopapular rashes of various sizes on the entire body, including the trunk and extremities. (C) The face affected with erythematous edema. 
od makes it difficult to diagnose the cause in many cas$\mathrm{es}^{4,11}$. In the present case, we presumed that lamotrigine, which had been administered for 3 weeks prior to the symptoms, was the causative drug. We reviewed a report that analyzed clinical data from 37 published reports with 57 cases diagnosed with DRESS syndrome or drug-induced hypersensitivity syndrome (DIHS) related to lamotrigine therapy. The cases were collected from published papers in PubMed and the Scopus database (from January 1999 to April 2014) ${ }^{13}$. In Korea, two cases of severe drug eruptions associated with lamotrigine treatment have been reported. One was a case of Stevens-Johnson syndrome induced by the combined use of lamotrigine and valproic acid $^{14}$. The clinical features of the other case met the criteria for DRESS syndrome, but the case was diagnosed as 'anticonvulsant hypersensitivity syndrome' ${ }^{\prime}$.

DRESS syndrome was first referred to as drug-hypersensitivity syndrome by Chaiken et al. ${ }^{15}$ in 1950, and both terms have been used by researchers; thus, there has been no consistent definition or terminology ${ }^{16}$. Although DRESS syndrome is characterized by skin rashes accompanied by fever, internal organ involvement, and eosinophilia on laboratory test results, it can have variable clinical manifestations on a patient-by-patient basis. A typical skin lesion includes erythematous, maculopapular rashes of diverse sizes over the entire body; they appear symmetrical, but they combine as they spread out. They also cause the patient to experience severe itchiness ${ }^{4,11,12}$. The rashes usually start on the facial area, upper body, and upper extremities, and then they move to the lower extremities. Unlike typical drug eruptions that do not involve the facial area, patients with DRESS syndrome frequently present with erythema and edema of the face ${ }^{11}$. About $50 \%$ to $60 \%$ of cases with organ involvement occur in the liver, and hepatic failure is a prognosis of DRESS syndrome. When the causative drug is discontinued after a short period after the symptom outbreak, the liver enzyme levels can quickly normalize. Alternatively, it may progress into fulminant hepatitis or hepatic enlargement; hepatic failure is a common cause of death in DRESS syndrome ${ }^{17}$. Also, there are rare cases in which the kidney, lung, or nervous system is involved ${ }^{1,2}$. In the present case, the patient had skin rashes that progressed from the trunk to the entire body, including the face, and the rashes were accompanied by a fever over $38^{\circ} \mathrm{C}$, increased liver enzyme levels, and significant eosinophilia (28\%). Thus, he was diagnosed with DRESS syndrome, based on the scoring system for classifying DRESS, validated by Kardaun et al. ${ }^{18}$ in 2007. According to this system, he scored 5, which is indicative of DRESS syndrome.

The key to managing DRESS syndrome is the immediate discontinuation of the causative drug, as in other common cases of drug eruptions. When organ involvement is detected, systemic steroid administration is important, and symptomatic control is also required to resolve itchiness ${ }^{17}$. In our case, since the patient was consulted for a skin rash of the trunk without a systemic symptom, conservative treatment was initiated by discontinuing the causative drug, according to the standard treatment for drug eruption. However, 2 days after discontinuing the drug, the patient developed a fever, and the skin rash spread over the entire body, including his face, and the itching was aggravated. Initially, he was considered to have an infection since he had a fever, but no evidence of infection was detected on examination. When he returned to the department of dermatology, the possibility of severe drug eruption with internal organ involvement was considered. After the laboratory results were ascertained, DRESS syndrome was finally diagnosed, and systemic steroid administration was started. Although it is known that the initial symptoms of the syndrome are usually a skin rash with fever ${ }^{10,13}$, as in our case, it may start as a simple drug eruption and then systemic symptoms may be detected later. Therefore, caution is required when making a diagnosis. In the case that a common causative drug is the cause, especially an aromatic anticonvulsant, discontinuation of the drug is insufficient. Dermatologists should more carefully observe and consider the possible prognosis of the patient. In addition, it is important for dermatologists to educate the affected patients when the symptoms suddenly become exacerbated or systemic symptoms develop, as they should visit the hospital immediately for further evaluation and to start intensive treatment.

As skin rashes appear on $90 \%$ of patients with DRESS syndrome and most of patients' major complaints are itchiness and skin rashes, dermatologists are involved in the diagnosis and treatment of most cases. The mortality of DRESS syndrome is $10 \%^{10-12}$, and this is mainly because of the involvement of systemic organs such as the liver. As mentioned previously, the degree of involvement is commensurate with the length of the period from symptom outbreak to the point of causative drug discontinuation; therefore, dermatologists should be highly aware of this disease.

We experienced a case of DRESS syndrome induced by lamotrigine, which is rare. The present case emphasizes that close observation of patients with drug eruption induced by an aromatic anticonvulsant is imperative. Dermatologists should be able to promptly diagnose patients with DRESS syndrome, detect the causative drug, immediately discontinue the causative drug, and play a crucial role in the patient's evaluation and treatment. 


\section{CONFLICTS OF INTEREST}

The authors have nothing to disclose.

\section{REFERENCES}

1. Lee JE, Goo BC, Kim YC, Lee JH, Lee $\mathrm{KH}$. A case of anticonvulsant hypersensitivity syndrome thought to be caused by lamotrigine. Korean J Dermatol 2006;44:620623.

2. Knowles SR, Shapiro LE, Shear NH. Anticonvulsant hypersensitivity syndrome: incidence, prevention and management. Drug Saf 1999;21:489-501.

3. Jeon EK, Lee KM, Kwon YB, Seo YJ, Park JK, Lee JH. Three cases of allopurinol-induced DRESS syndrome. Korean J Dermatol 2007;45:714-719.

4. Tas S, Simonart T. Management of drug rash with eosinophilia and systemic symptoms (DRESS syndrome): an update. Dermatology 2003;206:353-356.

5. Ichiche $M$, Kiesch N, De Bels D. DRESS syndrome associated with HHV-6 reactivation. Eur J Intern Med 2003; 14:498-500.

6. Kim $\mathrm{TI}$, Jeong $\mathrm{KH}$, Shin MK, Kim NI. Piperacillin/Tazobactam-Associated hypersensitivity syndrome with overlapping features of acute generalized exanthematous pustulosis and drug-related rash with eosinophilia and systemic symptoms syndrome. Ann Dermatol 2016;28:98-101.

7. Schlienger RG, Knowles SR, Shear NH. Lamotrigine-associated anticonvulsant hypersensitivity syndrome. Neurology 1998;51:1172-1175.

8. Beller TC, Boyce JA. Prolonged anticonvulsant hypersensitivity syndrome related to lamotrigine in a patient with human immunodeficiency virus. Allergy Asthma Proc 2002;23:415419.
9. Fervenza FC, Kanakiriya S, Kunau RT, Gibney R, Lager DJ. Acute granulomatous interstitial nephritis and colitis in anticonvulsant hypersensitivity syndrome associated with lamotrigine treatment. Am J Kidney Dis 2000;36:1034-1040.

10. Kim YH, Choi CW, Lee GY, Kim WS. A case of valproic acid induced DRESS syndrome. Korean J Dermatol 2012; 50:85-88

11. Bachot N, Roujeau JC. Differential diagnosis of severe cutaneous drug eruptions. Am J Clin Dermatol 2003;4: 561-572.

12. Bessmertny O, Pham T. Antiepileptic hypersensitivity syndrome: clinicians beware and be aware. Curr Allergy Asthma Rep 2002;2:34-39.

13. Wang $X Q$, Lv B, Wang HF, Zhang $X, Y u$ SY, Huang XS, et al. Lamotrigine induced DIHS/DRESS: Manifestations, treatment, and outcome in 57 patients. Clin Neurol Neurosurg 2015;138:1-7.

14. Kim JW, Kim KS. Two cases of stevens-johnson syndrome related to concomitant use of lamotrigine and valproic acid. Korean J Dermatol 2005;43:1105-1109.

15. Chaiken BH, Goldberg BI, Segal JP. Dilantin sensitivity; report of a case of hepatitis with jaundice, pyrexia and exfoliative dermatitis. N Engl J Med 1950;242:897-898.

16. Sontheimer RD, Houpt KR. DIDMOHS: a proposed consensus nomenclature for the drug-induced delayed multiorgan hypersensitivity syndrome. Arch Dermatol 1998;134:874876.

17. Roujeau JC. Treatment of severe drug eruptions. J Dermatol 1999;26:718-722.

18. Kardaun SH, Sidoroff A, Valeyrie-Allanore L, Halevy S, Davidovici BB, Mockenhaupt $M$, et al. Variability in the clinical pattern of cutaneous side-effects of drugs with systemic symptoms: does a DRESS syndrome really exist? $\mathrm{Br}$ J Dermatol 2007;156:609-611. 